ACC 2013

\section{Mekka der Herzmediziner}

Alljährlich im März findet der Jahreskongress des „American College of Cardiology“ (ACC) statt, einer der drei großen internationalen Kongresse der Herzmedizin. Wenngleich sich die transatlantischen Gewichte verschoben haben - der europäische Kardiologenkongress ist heute von Bedeutung und Teilnehmerzahl her die klare Nummer 1 - bietet der ACC doch immer noch eine Fülle neuer Erkenntnisse. Mehr als 20 sog. „Late Breaking Clinical Trials" wurden vorgestellt. Hier auf Seite 1 lesen Sie das Wichtigste in Kürze. In der nächsten Ausgabe Ihrer MMW berichten wir ausführlicher über das, was für die niedergelassene Praxis relevant ist.

\section{VORHOFFLIMMERN}

\section{Herzohrverschluss statt Antikoagulation?}

Wenn Patienten mit Vorhofflimmern ein hinreichend hohes Schlaganfall-Risiko aufweisen (CHA2DS2-VASc-Score von mindestens 2), ist eine Antikoagulation indiziert. Doch viele Patienten wollen oder können nicht zuverlässig antikoagulieren. Für diese Patienten gibt es eine interventionelle Behandlungsalternative: Ein transseptal in den linken Vorhof eingebrachtes Schirmchen, welches das linke Herzohr abriegelt und so verhindert, dass Thromben von dort embolisiert werden. Wirksamkeit und Sicherheit des sog. WATCHMAN-Device sind nun bereits in zwei Studien mit der oralen Antikoagulation verglichen worden: Die Ergebnisse sind gut und die Komplikationsrate sinkt.

\section{AORTENKLAPPENSTENOSE}

\section{Prognose nach TAVI-Eingriff immer besser}

Mit dem Aufkommen des interventionellen Aortenklappen-Ersatzes vor wenigen Jahren hat sich die Therapie der schweren Aortenklappenstenose bei hochbetagten Patienten revolutioniert. Für inoperable Patienten bietet der Eingriff eine Lebensverlängerung, für Patienten mit hohem Operati- onsrisiko eine schonende Alternative. Neue Daten bestätigen nun, dass die Sterblichkeit nach dem Eingriff mit zunehmender Erfahrung deutlich niedriger liegt als noch vor einigen Jahren. Auch die Klappensysteme werden technisch weiter verbessert und kleiner, der Eingriff damit noch schonender.

\section{KORONARE HERZKRANKHEIT}

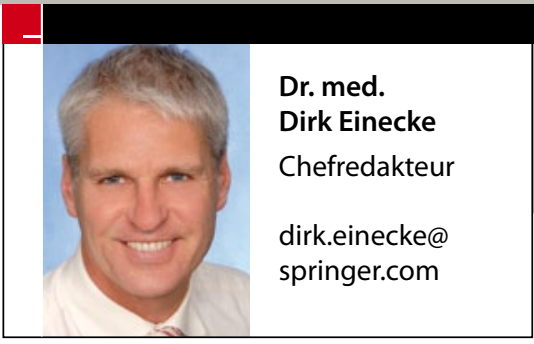

ST-HEBUNGS-HERZINFARKT

\section{Aldosteron-Blockade für alle von Vorteil?}

Die Behandlung mit Eplerenon hilft sowohl Patienten, die nach Herzinfarkt eine linksventrikuläre Dysfunktion aufweisen, als auch Patienten, die an einer chronischen Herzinsuffizienz leiden.

In der REMINDER-Studie wurde nun bei über 1000 Patienten geprüft, ob der Aldosteronhemmer nach Herzinfarkt grundsätzlich vorteilhaft ist, also auch dann, wenn der Patient keine Herzschwäche entwickelt. Die Studie ging scheinbar gut aus, das relative Risiko für den primären Studienendpunkt wurde um $38 \%$ reduziert.

Ein genauer Blick zeigt jedoch, dass v.a. die Werte für natriuretische Peptidhormone reduziert werden, aber keine klinischen Endpunkte. Erhöhte BNP/NT-proBNP-Werte gelten als Marker für eine schlechtere Prognose.

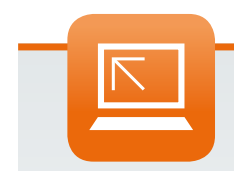

ACC auf springermedizin.de

Ausführliche Kongressberichte vom amerikanischen Kardiologenkongress finden Sie online unter.

noch knapp sieben Angina-Attacken in der Der selektive Inhibitor des späten NatriumEinstroms ( $\mathrm{I}_{\mathrm{Na}}$ Late) Ranolazin kann bei chronisch koronarkranken Diabetikern mit unzureichend behandelter Angina pectoris die Beschwerdesymptomatik reduzieren. Dies zeigte die sog. TERISA-Studie mit über 900 Patienten. Hatten die Patienten zuvor
Woche, so sank die Frequenz unter der Therapie auf unter vier. Ein genauer Blick aber zeigt: Der Großteil der Verbesserung ließ sich auch mit Plazebo erreichen, obgleich der Unterschied zwischen Verum und Plazebo statistisch signifikant war. rwww.

springer-

medizin.de/

acc-2013

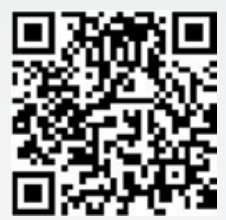

